Supporting Information for

\title{
Probing a silent metal: A Combined X-ray Absorption and Emission Spectroscopic Study of Biologically Relevant Zinc Complexes
}

\author{
Olivia McCubbin Stepanic, ${ }^{\mathrm{a}}$ Jesse Ward, ${ }^{\mathrm{b}, \mathrm{d}}$ James E. Penner-Hahn, ${ }^{\mathrm{b}}$ Aniruddha Deb, ${ }^{\mathrm{b}}$ Uwe \\ Bergmann, ${ }^{c *}$ Serena DeBeer ${ }^{*}$ \\ ${ }^{a}$ Max Planck Institute for Chemical Energy Conversion, Stiftstr. 34-36, D-45470 Mülheim an der Ruhr, Germany \\ ${ }^{b}$ University of Michigan, Ann Arbor, Michigan 48109, United States \\ 'PULSE Institute, SLAC National Accelerator Laboratory, Menlo Park, California 94025, United States \\ ${ }^{\mathrm{d} C}$ Current address: PNNL, Richland, Washington 99354 United States \\ email: bergmann@slac.stanford.edu; serena.debeer@cec.mpg.de
}

\section{Table of Contents}

$\begin{array}{ll}\text { 1. Sample Input Files for Calculations } & \text { p. } 2\end{array}$

2. Optimized Molecular Geometries
a. $\mathrm{Zn}(\mathrm{BzO})_{2}\left(\mathrm{SC}\left(\mathrm{NH}_{2}\right)_{2}\right)_{2}$
p. 2
b. $\mathrm{Zn}(\mathrm{BzO})_{2}\left(\mathrm{pyNH}_{2}\right)_{2}$
p. 3
c. $\mathrm{Zn}(\mathrm{SPh})_{4}$
p. 4
d. $\mathrm{Zn}\left({ }^{\mathrm{Me}} \mathrm{Im}\right)_{2}(\mathrm{SPh})_{2}$
p. 5
e. $\mathrm{Zn}\left({ }^{\mathrm{Me}} \mathrm{Im}\right)_{4}$
p. 6
f. $\mathrm{Zn}\left({ }^{\mathrm{Me}} \mathrm{Im}\right)_{6}$
p. 8

VtC XES data collection from low to high and high to low energy: Figure S1 p. 10

3. Experimental VtC XES Fits: Figure S2

p. 13

4. K $\beta$ Mainline Overlay: Figure S3

p. 16

5. $\mathrm{Zn}\left({ }^{\mathrm{Me}} \mathrm{Im}\right)_{2}(\mathrm{SPh})_{2}$ Ligand-Ligand Interaction Calculations: Figure $\mathrm{S} 4$

p. 17

a. Molecular model of $\mathrm{Zn}\left({ }^{\mathrm{Me}} \mathrm{Im}\right)_{2}(\mathrm{SPh})_{2}$

b. Zn-S-C Angle Modulation Impact on VtC Spectra 


$\begin{array}{llcc}6 & 1.694358000 & -0.688108000 & 7.139096000 \\ 1 & 1.975637000 & 0.345998000 & 6.994866000 \\ 30 & -3.300237000 & 0.987901000 & 8.040394000 \\ 8 & -0.301568000 & 1.283951000 & 7.193385000 \\ 16 & -2.436428000 & 2.210764000 & 9.908130000 \\ 6 & -3.585938000 & 3.433659000 & 10.240529000 \\ 7 & -4.868762000 & 3.346587000 & 9.955611000 \\ 7 & -3.137636000 & 4.571701000 & 10.826865000 \\ 1 & -5.474258000 & 4.135629000 & 10.118788000 \\ 1 & -5.306779000 & 2.478103000 & 9.570407000 \\ 1 & -2.173317000 & 4.593900000 & 11.110414000 \\ 1 & -3.776499000 & 5.171863000 & 11.322751000 \\ 16 & -4.111149000 & 2.574847000 & 6.456524000 \\ 6 & -2.869491000 & 3.735028000 & 6.269327000 \\ 7 & -3.228778000 & 4.972279000 & 5.843138000 \\ 1 & -2.542453000 & 5.577075000 & 5.421388000 \\ 1 & -4.184321000 & 5.098921000 & 5.557508000 \\ 7 & -1.596572000 & 3.510469000 & 6.516321000 \\ 1 & -1.215890000 & 2.582681000 & 6.817189000 \\ 1 & -0.932056000 & 4.265355000 & 6.443760000\end{array}$

\section{d. $\mathrm{Zn}(\mathrm{BzO})_{2}\left(\mathrm{pyNH}_{2}\right)_{2}$}

$\begin{array}{cccc}8 & -4.634485000 & -0.205016000 & 7.286244000 \\ 6 & -5.541515000 & 0.008825000 & 8.136391000 \\ 6 & -6.819117000 & -0.764049000 & 8.091543000 \\ 6 & -6.981767000 & -1.785948000 & 7.145064000 \\ 1 & -6.157682000 & -1.997941000 & 6.463473000 \\ 6 & -8.174292000 & -2.507108000 & 7.090226000 \\ 1 & -8.296151000 & -3.303368000 & 6.354053000 \\ 6 & -9.214516000 & -2.209651000 & 7.976991000 \\ 1 & -10.148626000 & -2.772033000 & 7.931076000 \\ 6 & -9.057332000 & -1.191046000 & 8.922102000 \\ 1 & -9.869123000 & -0.957079000 & 9.612979000 \\ 6 & -7.862986000 & -0.472325000 & 8.981049000 \\ 1 & -7.720304000 & 0.327127000 & 9.707950000 \\ 8 & -5.394401000 & 0.909777000 & 9.051788000 \\ 1 & -4.790360000 & 1.391930000 & 10.683184000 \\ 7 & -3.979402000 & 2.777697000 & 6.850496000 \\ 6 & -3.573046000 & 2.735608000 & 5.554596000 \\ 6 & -4.237113000 & 3.511488000 & 4.570320000 \\ 1 & -3.903478000 & 3.459541000 & 3.533390000 \\ 6 & -5.290123000 & 4.323225000 & 4.943356000 \\ 1 & -5.806328000 & 4.920846000 & 4.190633000 \\ 6 & -5.690857000 & 4.374981000 & 6.289323000\end{array}$




$\begin{array}{lccc}1 & -6.518179000 & 5.000702000 & 6.618613000 \\ 6 & -5.007250000 & 3.582825000 & 7.194346000 \\ 1 & -5.285111000 & 3.552281000 & 8.248904000 \\ 7 & -4.332947000 & 1.955617000 & 11.410482000 \\ 1 & -4.574775000 & 1.806975000 & 12.379873000 \\ 8 & -2.209825000 & -0.204999000 & 9.372111000 \\ 6 & -1.302798000 & 0.008846000 & 8.521964000 \\ 6 & -0.025191000 & -0.764020000 & 8.566815000 \\ 6 & 0.137462000 & -1.785917000 & 9.513296000 \\ 1 & -0.686624000 & -1.997912000 & 10.194885000 \\ 6 & 1.329990000 & -2.507072000 & 9.568137000 \\ 1 & 1.451851000 & -3.303330000 & 10.304312000 \\ 6 & 2.370214000 & -2.209612000 & 8.681375000 \\ 1 & 3.304327000 & -2.771989000 & 8.727293000 \\ 6 & 2.213029000 & -1.191008000 & 7.736262000 \\ 1 & 3.024819000 & -0.957039000 & 7.045387000 \\ 6 & 1.018679000 & -0.472292000 & 7.677311000 \\ 1 & 0.875995000 & 0.327158000 & 6.950409000 \\ 30 & -3.422165000 & 1.372909000 & 8.329175000 \\ 7 & -2.864921000 & 2.777690000 & 9.807861000 \\ 6 & -3.271328000 & 2.735639000 & 11.103744000 \\ 6 & -1.153404000 & 4.374889000 & 10.369074000 \\ 1 & -0.326037000 & 5.000563000 & 10.039809000 \\ 6 & -1.837020000 & 3.582759000 & 9.464035000 \\ 1 & -1.559125000 & 3.552190000 & 8.409486000 \\ 8 & -1.449920000 & 0.909791000 & 7.606560000 \\ 7 & -2.511480000 & 1.955528000 & 5.247827000 \\ 1 & -2.054044000 & 1.391851000 & 5.975120000 \\ 1 & -2.269693000 & 1.806869000 & 4.278429000 \\ 6 & -2.607260000 & 3.511498000 & 12.088035000 \\ 1 & -2.940937000 & 3.459585000 & 13.124954000 \\ 6 & -1.554193000 & 4.323174000 & 11.715026000 \\ 1 & -1.037984000 & 4.920779000 & 12.467759000\end{array}$

\section{e. $\mathrm{Zn}(\mathrm{SPh})_{4}$}

$\begin{array}{lccc}30 & -0.929808000 & 0.789444000 & -0.669967000 \\ 16 & -0.623285000 & 1.795111000 & 1.490317000 \\ 16 & -0.097782000 & -1.397379000 & -0.120825000 \\ 16 & -3.133011000 & 0.378770000 & -1.488607000 \\ 16 & 0.108001000 & 1.991089000 & -2.450389000 \\ 6 & 1.712729000 & 2.423517000 & -1.880778000 \\ 6 & 2.408188000 & 3.477003000 & -2.502377000 \\ 6 & 2.350678000 & 1.770859000 & -0.811510000 \\ 6 & 3.669799000 & 3.864187000 & -2.073797000 \\ 1 & 1.926463000 & 4.001316000 & -3.318859000\end{array}$




$\begin{array}{lrrr}6 & 3.608498000 & 2.168034000 & -0.382287000 \\ 1 & 1.843674000 & 0.958094000 & -0.311011000 \\ 6 & 4.284961000 & 3.214232000 & -1.006251000 \\ 1 & 4.173775000 & 4.686671000 & -2.572265000 \\ 1 & 4.062765000 & 1.651461000 & 0.456471000 \\ 1 & 5.258366000 & 3.533776000 & -0.651213000 \\ 6 & -3.771317000 & 1.941052000 & -1.969570000 \\ 6 & -4.785294000 & 2.019537000 & -2.943277000 \\ 6 & -3.324735000 & 3.153568000 & -1.414787000 \\ 6 & -5.307686000 & 3.239565000 & -3.350903000 \\ 1 & -5.144066000 & 1.099518000 & -3.389362000 \\ 6 & -3.842479000 & 4.370215000 & -1.828532000 \\ 1 & -2.558466000 & 3.133649000 & -0.654074000 \\ 6 & -4.838769000 & 4.430942000 & -2.802020000 \\ 1 & -6.081800000 & 3.261355000 & -4.112607000 \\ 1 & -3.453325000 & 5.278678000 & -1.381308000 \\ 1 & -5.236701000 & 5.384128000 & -3.133165000 \\ 6 & -0.097583000 & -2.330393000 & -1.607834000 \\ 6 & -0.040195000 & -1.742694000 & -2.883593000 \\ 6 & -0.133012000 & -3.736826000 & -1.549614000 \\ 6 & -0.030219000 & -2.521920000 & -4.030249000 \\ 1 & -0.000866000 & -0.664171000 & -2.965670000 \\ 6 & -0.117596000 & -4.511874000 & -2.702066000 \\ 1 & -0.187368000 & -4.210155000 & -0.575860000 \\ 6 & -0.067400000 & -3.913746000 & -3.958779000 \\ 1 & 0.005455000 & -2.028139000 & -4.995964000 \\ 1 & -0.154242000 & -5.594424000 & -2.616160000 \\ 1 & -0.064557000 & -4.516512000 & -4.859998000 \\ 6 & -0.548416000 & 3.548884000 & 1.473878000 \\ 6 & -0.644701000 & 4.242880000 & 2.697817000 \\ 6 & -0.386033000 & 4.327567000 & 0.313966000 \\ 6 & -0.597507000 & 5.628329000 & 2.755280000 \\ 1 & -0.767796000 & 3.665965000 & 3.606944000 \\ 6 & -0.345396000 & 5.713677000 & 0.377760000 \\ 1 & -0.292430000 & 3.834523000 & -0.642922000 \\ 6 & -0.452792000 & 6.385157000 & 1.593568000 \\ 1 & -0.678744000 & 6.124925000 & 3.718051000 \\ 1 & -0.219850000 & 6.274377000 & -0.542498000 \\ 1 & -0.419629000 & 7.468579000 & 1.636898000\end{array}$

\section{f. $\mathrm{Zn}\left({ }^{\mathrm{Me}} \mathrm{Im}\right)_{2}(\mathrm{SPh})_{2}$}

$\begin{array}{llll}30 & -0.428805000 & 3.632691000 & 2.293323000 \\ 16 & -1.475704000 & 2.605861000 & 0.507416000 \\ 7 & -1.872363000 & 4.838838000 & 3.181948000 \\ 7 & -3.947208000 & 5.387754000 & 3.635480000\end{array}$




$\begin{array}{lrrr}6 & -3.019159000 & 2.201394000 & 1.279384000 \\ 6 & -4.214078000 & 2.350713000 & 0.562919000 \\ 6 & -5.445397000 & 2.135214000 & 1.170489000 \\ 6 & -5.516814000 & 1.765756000 & 2.509990000 \\ 6 & -4.335256000 & 1.587933000 & 3.223427000 \\ 6 & -3.103028000 & 1.784933000 & 2.615609000 \\ 6 & -1.958177000 & 5.131240000 & 4.524624000 \\ 6 & -3.244815000 & 5.470649000 & 4.821876000 \\ 6 & -3.081909000 & 5.003467000 & 2.677812000 \\ 6 & -5.382101000 & 5.533510000 & 3.476071000 \\ 1 & -4.166385000 & 2.664186000 & -0.472214000 \\ 1 & -6.354098000 & 2.262588000 & 0.593854000 \\ 1 & -6.475451000 & 1.600833000 & 2.985537000 \\ 1 & -4.369323000 & 1.281748000 & 4.261996000 \\ 1 & -2.193334000 & 1.632570000 & 3.181962000 \\ 1 & -1.099505000 & 5.055771000 & 5.168631000 \\ 1 & -3.718213000 & 5.759513000 & 5.743254000 \\ 1 & -3.365752000 & 4.809570000 & 1.658491000 \\ 1 & -5.618608000 & 5.557086000 & 2.415336000 \\ 1 & -5.717784000 & 6.459223000 & 3.942429000 \\ 1 & -5.896856000 & 4.686035000 & 3.927552000 \\ 16 & 0.618374000 & 2.602254000 & 4.077099000 \\ 7 & 1.017113000 & 4.838193000 & 1.407554000 \\ 6 & 2.159007000 & 2.194814000 & 3.300877000 \\ 6 & 1.105041000 & 5.132825000 & 0.065526000 \\ 6 & 2.226097000 & 5.000802000 & 1.913653000 \\ 6 & 3.356040000 & 2.340542000 & 4.014548000 \\ 6 & 2.238595000 & 1.780037000 & 1.963856000 \\ 6 & 2.392431000 & 5.471468000 & -0.229381000 \\ 1 & 0.247159000 & 5.059358000 & -0.579773000 \\ 7 & 3.093125000 & 5.385783000 & 0.957823000 \\ 1 & 2.508233000 & 4.804776000 & 2.933034000 \\ 6 & 4.585380000 & 2.123761000 & 3.403428000 \\ 1 & 3.311583000 & 2.652347000 & 5.050330000 \\ 6 & 3.468877000 & 1.581826000 & 1.352493000 \\ 1 & 1.327218000 & 1.630137000 & 1.399546000 \\ 1 & 2.867386000 & 5.761445000 & -1.149605000 \\ 6 & 4.527954000 & 5.529630000 & 1.119537000 \\ 6 & 4.652618000 & 1.756560000 & 2.063074000 \\ 1 & 5.495802000 & 2.248371000 & 3.977956000 \\ 1 & 3.499688000 & 1.277237000 & 0.313353000 \\ 1 & 4.763023000 & 5.550404000 & 2.180653000 \\ 1 & 4.865263000 & 6.456102000 & 0.655849000 \\ 1 & 5.042391000 & 4.682670000 & 0.666745000 \\ 1 & 5.609718000 & 1.590823000 & 1.584730000\end{array}$




\section{g. $\mathrm{Zn}\left({ }^{\mathrm{Me}} \mathrm{Im}\right)_{4}$}

$\begin{array}{lrrr}30 & 18.615736000 & 0.000019000 & -3.723859000 \\ 7 & 17.288612000 & -1.069184000 & -2.657174000 \\ 7 & 16.095597000 & -2.800368000 & -2.027176000 \\ 6 & 16.289721000 & -0.626645000 & -1.809895000 \\ 1 & 16.187011000 & 0.407893000 & -1.531737000 \\ 6 & 17.138148000 & -2.384863000 & -2.759487000 \\ 1 & 17.756998000 & -3.046356000 & -3.340945000 \\ 6 & 15.544604000 & -1.696603000 & -1.412975000 \\ 1 & 14.693806000 & -1.767627000 & -0.758227000 \\ 6 & 15.653914000 & -4.186829000 & -1.895083000 \\ 1 & 16.246351000 & -4.807272000 & -2.561632000 \\ 1 & 14.604149000 & -4.267246000 & -2.172756000 \\ 1 & 15.796282000 & -4.524377000 & -0.869746000 \\ 7 & 19.943525000 & 1.069019000 & -2.657759000 \\ 7 & 21.137531000 & 2.799896000 & -2.028781000 \\ 6 & 20.943171000 & 0.626203000 & -1.811515000 \\ 1 & 21.045946000 & -0.408381000 & -1.533553000 \\ 6 & 20.094167000 & 2.384674000 & -2.760095000 \\ 1 & 19.474875000 & 3.046360000 & -3.340857000 \\ 6 & 21.688903000 & 1.695965000 & -1.415221000 \\ 1 & 22.540349000 & 1.766761000 & -0.761293000 \\ 6 & 21.579471000 & 4.186288000 & -1.896839000 \\ 1 & 20.986981000 & 4.806810000 & -2.563268000 \\ 1 & 22.629179000 & 4.266519000 & -2.174779000 \\ 1 & 21.437419000 & 4.523893000 & -0.871476000 \\ 7 & 17.546415000 & 1.327533000 & -4.789991000 \\ 7 & 15.815119000 & 2.520723000 & -5.419349000 \\ 6 & 17.988837000 & 2.326842000 & -5.636834000 \\ 1 & 19.023351000 & 2.429751000 & -5.915007000 \\ 6 & 16.230730000 & 1.477842000 & -4.687563000 \\ 1 & 15.569303000 & 0.858677000 & -4.106369000 \\ 6 & 16.918818000 & 3.072102000 & -6.033318000 \\ 1 & 16.847706000 & 3.923221000 & -6.687638000 \\ 6 & 14.428615000 & 2.962330000 & -5.551213000 \\ 1 & 13.808267000 & 2.369654000 & -4.884786000 \\ 1 & 14.348134000 & 4.012009000 & -5.273232000 \\ 1 & 14.091003000 & 2.820230000 & -6.576565000 \\ 7 & 19.684685000 & -1.327260000 & -4.790694000 \\ 7 & 21.415861000 & -2.520240000 & -5.420807000 \\ 6 & 19.242079000 & -2.326370000 & -5.637678000 \\ 1 & 18.207493000 & -2.429248000 & -5.915597000 \\ 6 & 21.000403000 & -1.477583000 & -4.688619000 \\ 6 & 21.661965000 & -0.858574000 & -4.107415000 \\ & 20.312026000 & -3.071469000 & -6.034664000\end{array}$




$\begin{array}{llll}1 & 20.382993000 & -3.922407000 & -6.689237000 \\ 6 & 22.802332000 & -2.961850000 & -5.553024000 \\ 1 & 23.422829000 & -2.369259000 & -4.886662000 \\ 1 & 22.882858000 & -4.011562000 & -5.275178000 \\ 1 & 23.139734000 & -2.819641000 & -6.578431000\end{array}$

h. $\mathrm{Zn}\left({ }^{\mathrm{Me}} \mathrm{Im}\right)_{6}$

$\begin{array}{lccc}30 & 15.526614000 & 0.646268000 & -3.079572000 \\ 7 & 15.397531000 & -0.523604000 & -1.213433000 \\ 7 & 16.137095000 & -1.875288000 & 0.361300000 \\ 6 & 14.953519000 & -0.064381000 & 0.006821000 \\ 1 & 14.366748000 & 0.830977000 & 0.098756000 \\ 6 & 16.103682000 & -1.613788000 & -0.962963000 \\ 1 & 16.606058000 & -2.218706000 & -1.697301000 \\ 6 & 15.406247000 & -0.893496000 & 0.993315000 \\ 1 & 15.278565000 & -0.873646000 & 2.061809000 \\ 6 & 16.860509000 & -2.961827000 & 1.010685000 \\ 1 & 17.337108000 & -3.574110000 & 0.248242000 \\ 1 & 16.174603000 & -3.579884000 & 1.588328000 \\ 1 & 17.625382000 & -2.555814000 & 1.671676000 \\ 7 & 17.396419000 & 1.529720000 & -2.324015000 \\ 7 & 19.345765000 & 2.555936000 & -2.309954000 \\ 6 & 18.015869000 & 1.284212000 & -1.117812000 \\ 1 & 17.552098000 & 0.693045000 & -0.350067000 \\ 6 & 18.220582000 & 2.301665000 & -3.014120000 \\ 1 & 18.041816000 & 2.678848000 & -4.006181000 \\ 6 & 19.226433000 & 1.914230000 & -1.097689000 \\ 1 & 19.997422000 & 1.967806000 & -0.348705000 \\ 6 & 20.493652000 & 3.343988000 & -2.742395000 \\ 1 & 20.306160000 & 3.725958000 & -3.743094000 \\ 1 & 21.387047000 & 2.720767000 & -2.755761000 \\ 1 & 20.648978000 & 4.182151000 & -2.065713000 \\ 7 & 13.656724000 & -0.236849000 & -3.837047000 \\ 7 & 11.708394000 & -1.265037000 & -3.852877000 \\ 6 & 13.036824000 & 0.010419000 & -5.042671000 \\ 1 & 13.499893000 & 0.603454000 & -5.809391000 \\ 6 & 12.833437000 & -1.011006000 & -3.148373000 \\ 1 & 13.012746000 & -1.390220000 & -2.157184000 \\ 6 & 11.826838000 & -0.620674000 & -5.063810000 \\ 1 & 11.055796000 & -0.673567000 & -5.812795000 \\ 6 & 10.561351000 & -2.055112000 & -3.421922000 \\ 1 & 10.749347000 & -2.439018000 & -2.422062000 \\ 1 & 9.667359000 & -1.432782000 & -3.407104000 \\ 1 & 10.406762000 & -2.891995000 & -4.100359000 \\ 7 & 16.666564000 & -0.959219000 & -4.031694000\end{array}$




$\begin{array}{llll}7 & 18.390384000 & -2.084475000 & -4.813287000 \\ 6 & 16.198668000 & -1.992052000 & -4.814660000 \\ 1 & 15.147399000 & -2.151312000 & -4.976168000 \\ 6 & 17.985879000 & -1.045980000 & -4.053689000 \\ 1 & 18.668110000 & -0.392139000 & -3.540016000 \\ 6 & 17.259930000 & -2.696903000 & -5.309307000 \\ 1 & 17.309821000 & -3.558315000 & -5.952151000 \\ 6 & 19.774471000 & -2.450834000 & -5.087301000 \\ 1 & 20.426368000 & -1.839006000 & -4.469168000 \\ 1 & 19.938937000 & -3.499510000 & -4.844906000 \\ 1 & 20.010992000 & -2.274443000 & -6.136334000 \\ 1 & 15.907718000 & 3.454472000 & -1.200757000 \\ 6 & 14.856164000 & 3.293717000 & -1.358869000 \\ 6 & 13.795789000 & 4.004057000 & -0.870218000 \\ 1 & 13.746975000 & 4.872182000 & -0.236427000 \\ 1 & 11.118375000 & 4.804181000 & -1.326026000 \\ 7 & 14.386822000 & 2.252685000 & -2.130046000 \\ 7 & 12.664495000 & 3.386840000 & -1.358106000 \\ 6 & 13.067591000 & 2.339960000 & -2.106971000 \\ 6 & 11.280818000 & 3.755048000 & -1.084391000 \\ 1 & 12.384286000 & 1.680734000 & -2.612160000 \\ 1 & 10.628201000 & 3.144998000 & -1.703522000 \\ 1 & 11.043329000 & 3.578246000 & -0.035618000 \\ 1 & 13.421573000 & 3.843462000 & -7.832840000 \\ 1 & 14.442236000 & 3.510429000 & -4.463992000 \\ 1 & 13.707080000 & 4.862828000 & -6.409602000 \\ 6 & 14.946092000 & 2.906384000 & -5.198032000 \\ 6 & 14.185346000 & 4.251714000 & -7.171945000 \\ 7 & 14.911743000 & 3.167318000 & -6.522399000 \\ 7 & 15.655328000 & 1.818308000 & -4.947166000 \\ 6 & 15.645293000 & 2.187364000 & -7.154150000 \\ 6 & 16.100447000 & 1.359931000 & -6.167347000 \\ 1 & 15.772905000 & 2.167468000 & -8.222654000 \\ 1 & 14.869525000 & 4.871606000 & -7.749677000 \\ 1 & 16.689911000 & 0.466345000 & -6.259244000\end{array}$


a. $\mathrm{Zn}(\mathrm{BzO})_{2}\left(\mathrm{SC}\left(\mathrm{NH}_{2}\right)_{2}\right)_{2}$

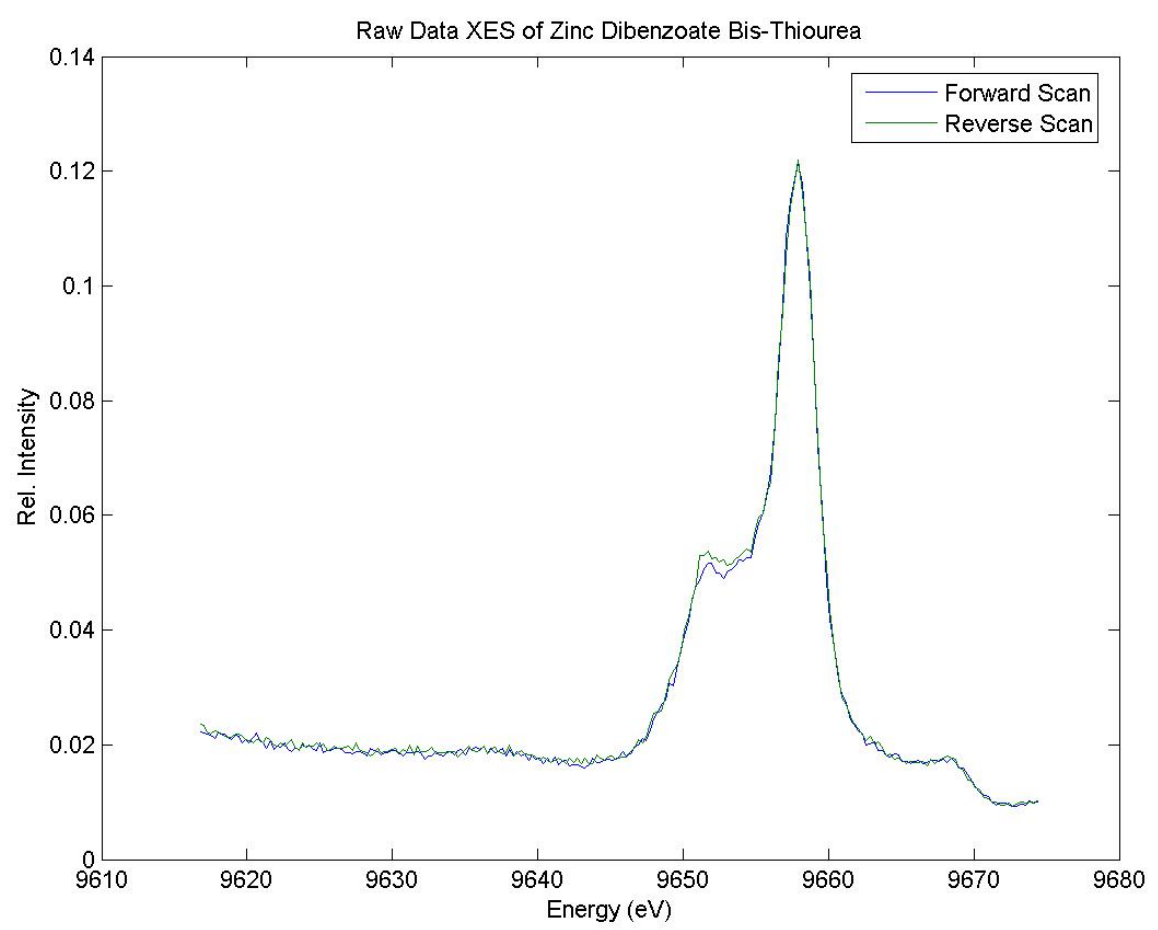

b. $\mathrm{Zn}(\mathrm{BzO})_{2}\left(\mathrm{pyNH}_{2}\right)_{2}$

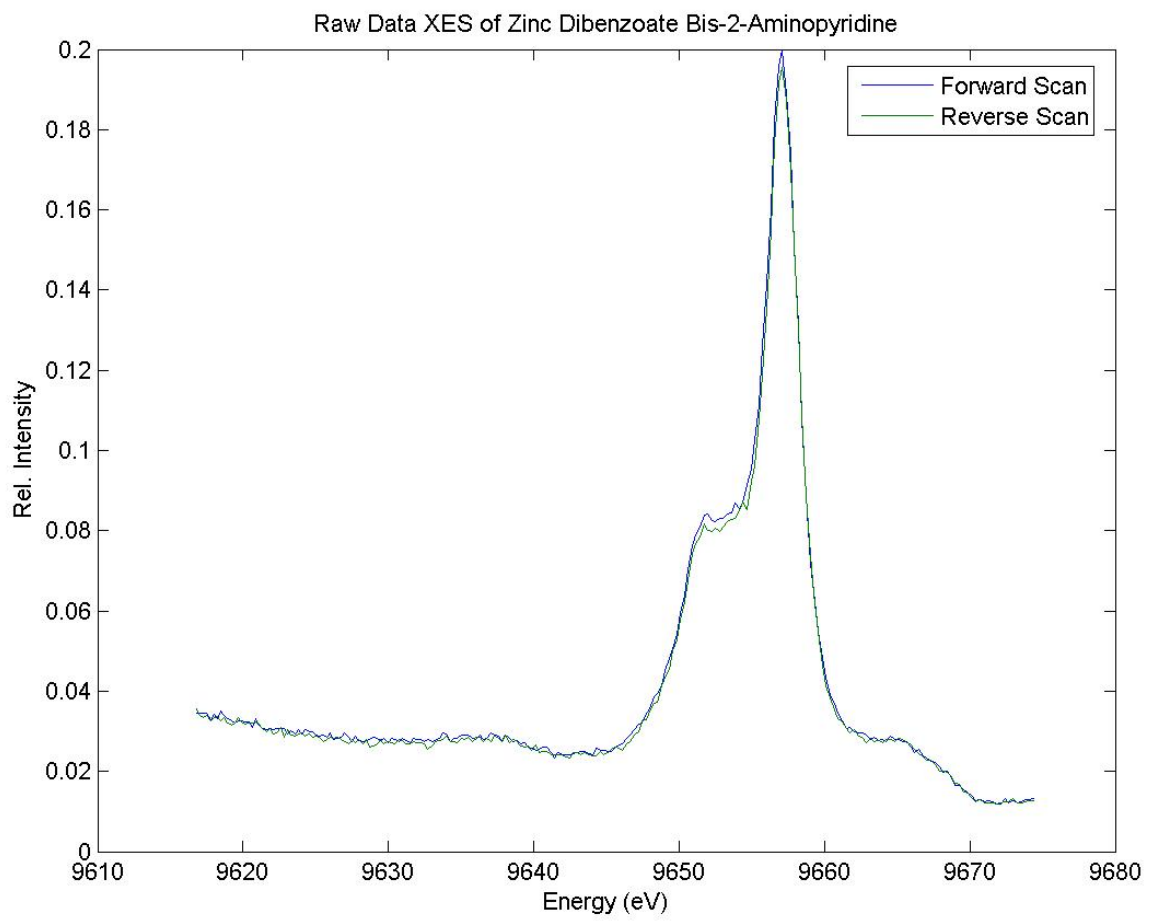




\section{c. $\mathrm{Zn}(\mathrm{SPh})_{4}$}

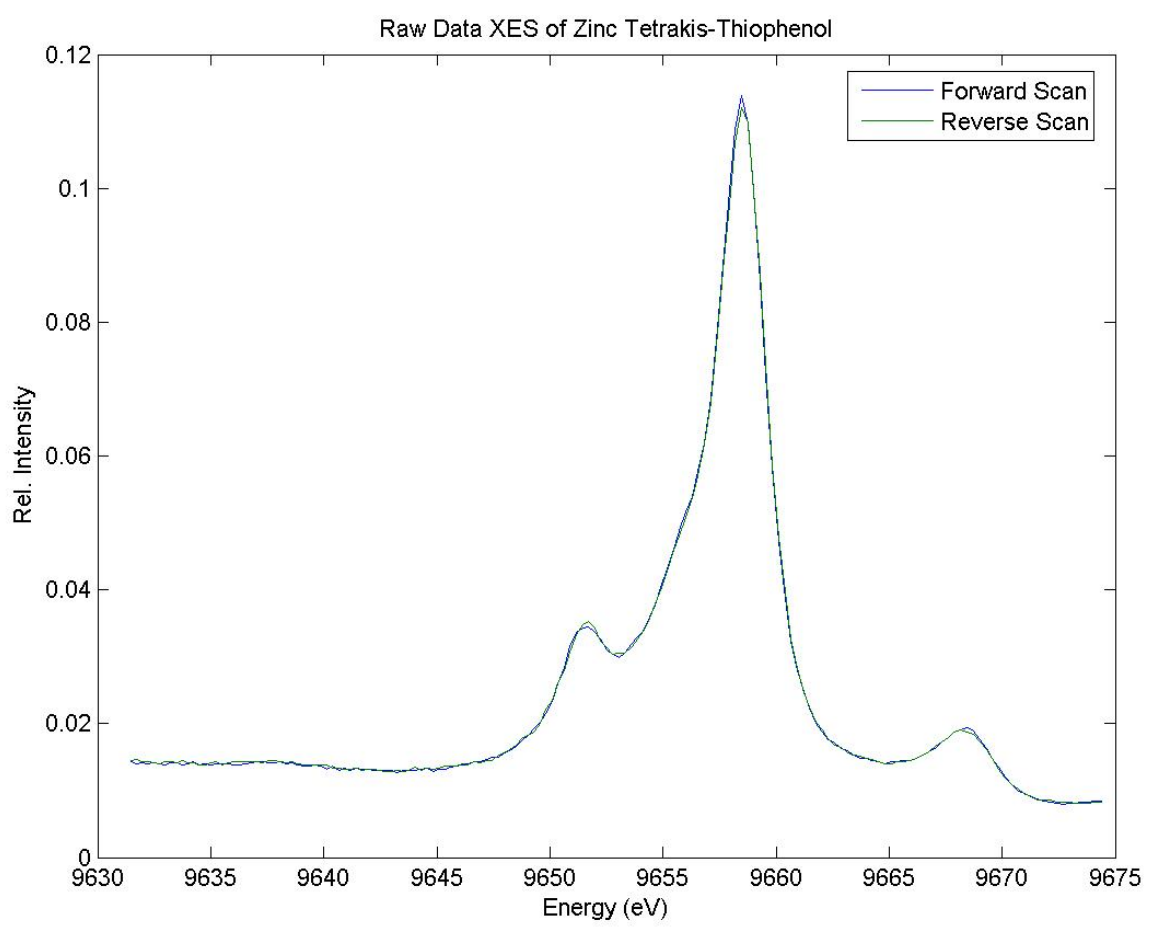

d. $\mathrm{Zn}\left({ }^{\mathrm{Me}} \mathrm{Im}\right)_{2}(\mathrm{SPh})_{2}$

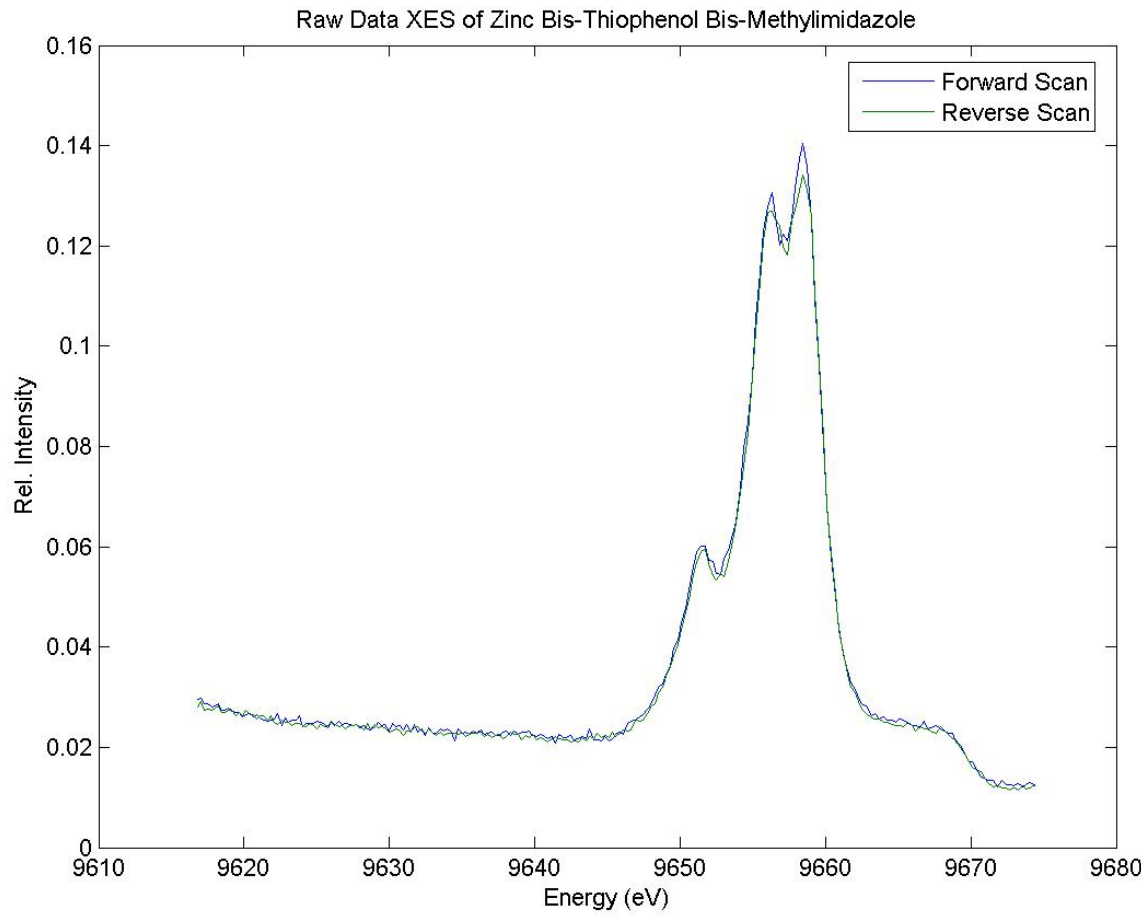


e. $\mathrm{Zn}\left({ }^{\mathrm{Me}} \mathrm{Im}\right)_{4}$

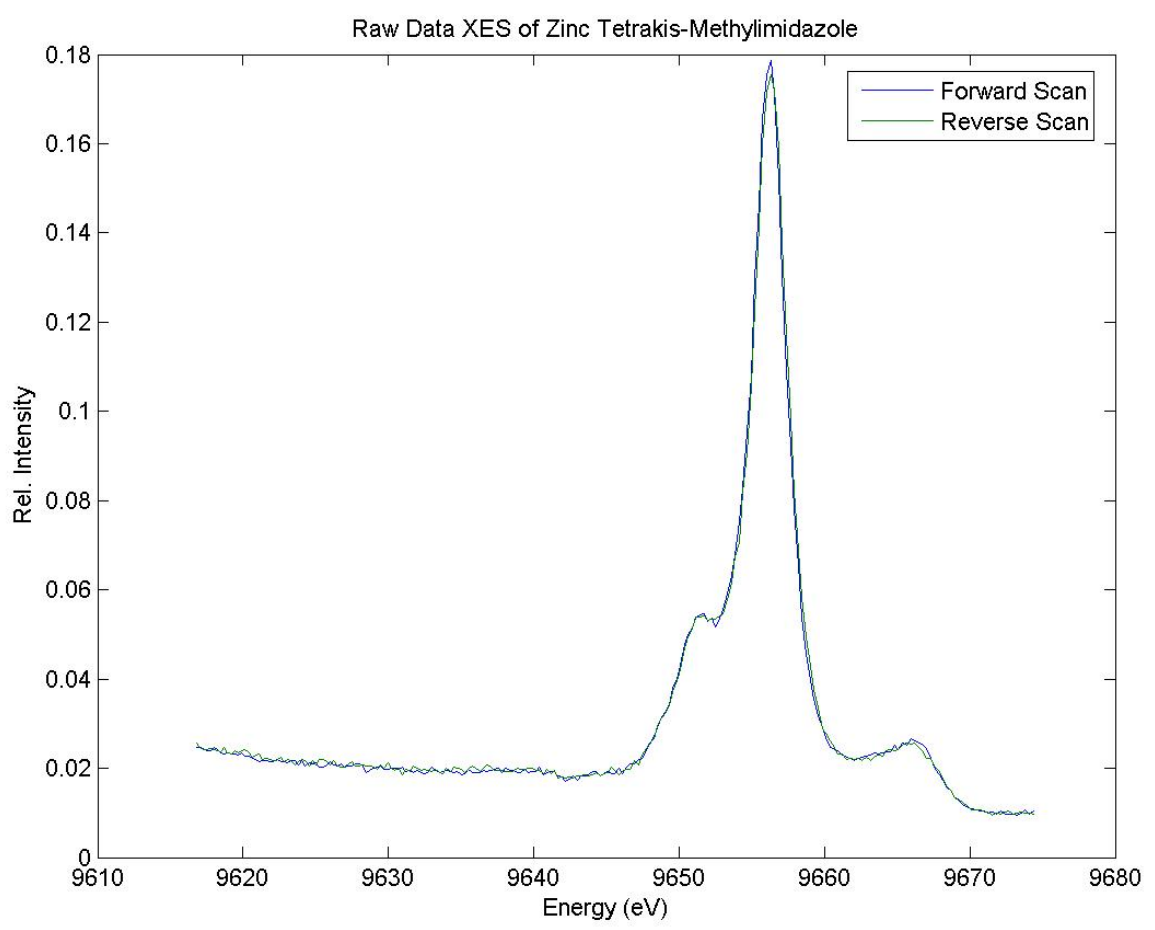

f. $\mathrm{Zn}\left({ }^{\mathrm{Me}} \mathrm{Im}\right)_{6}$

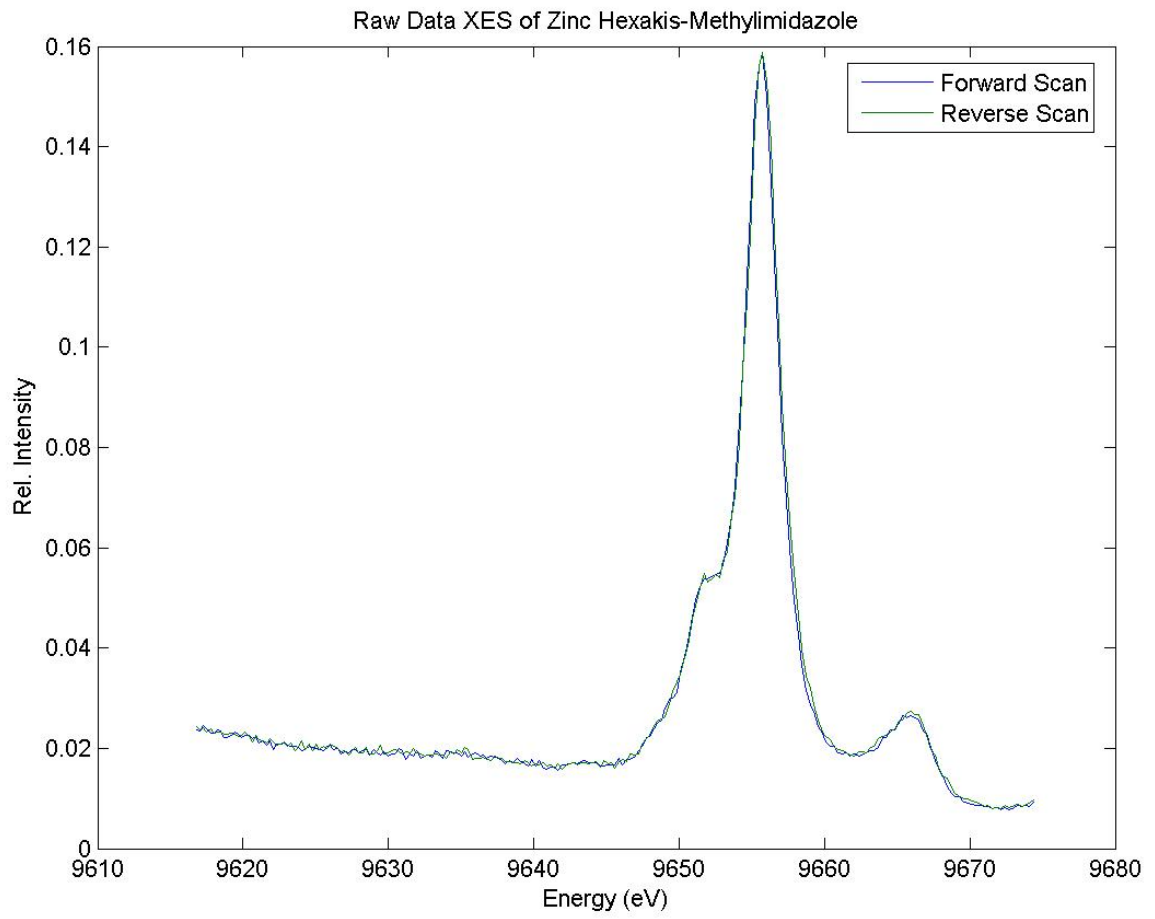

Figure S1. Zn VtC XES cans were collected from both lowest to highest energy and highest to lowest energy. The forward and reverse scans are shown for each complex. 
a. $\mathrm{Zn}(\mathrm{BzO})_{2}\left(\mathrm{SC}\left(\mathrm{NH}_{2}\right)_{2}\right)_{2}$

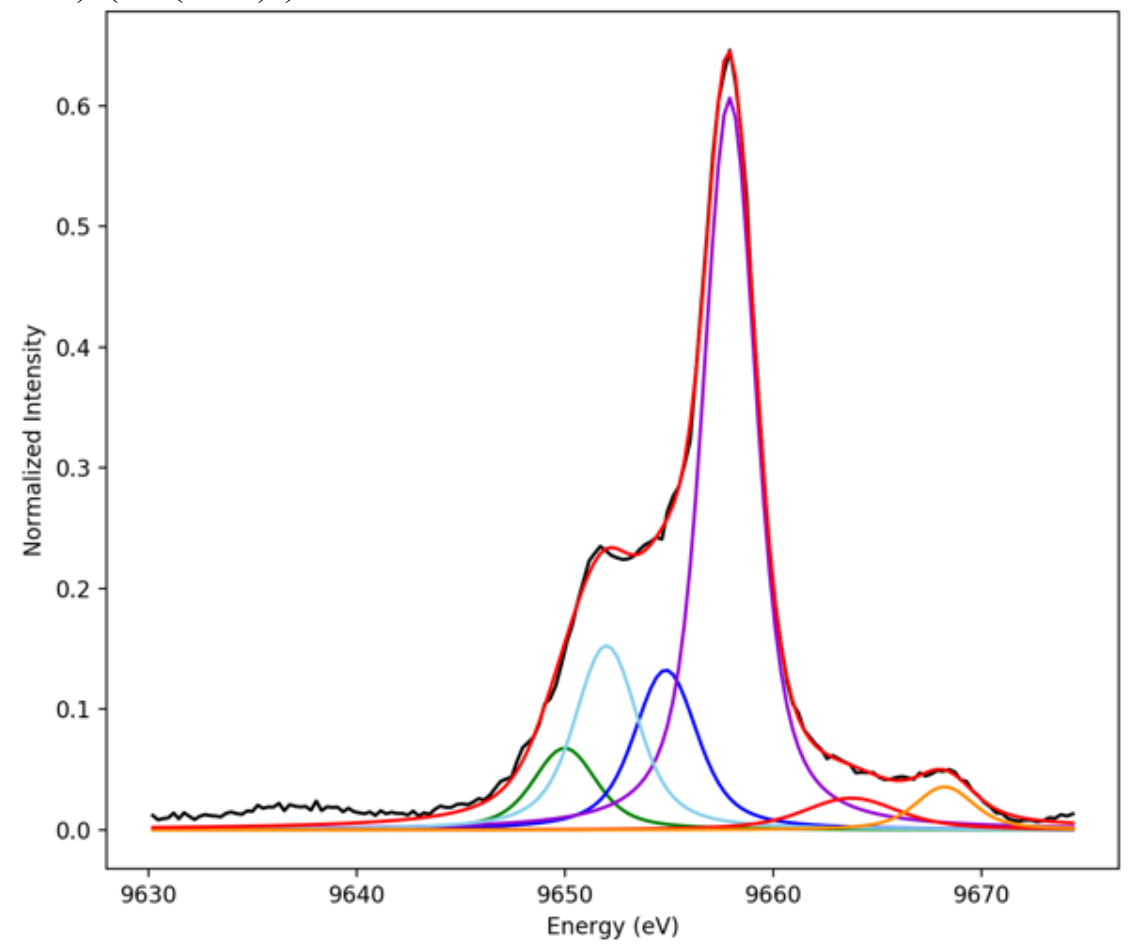

b. $\mathrm{Zn}(\mathrm{BzO})_{2}\left(\mathrm{pyNH}_{2}\right)_{2}$

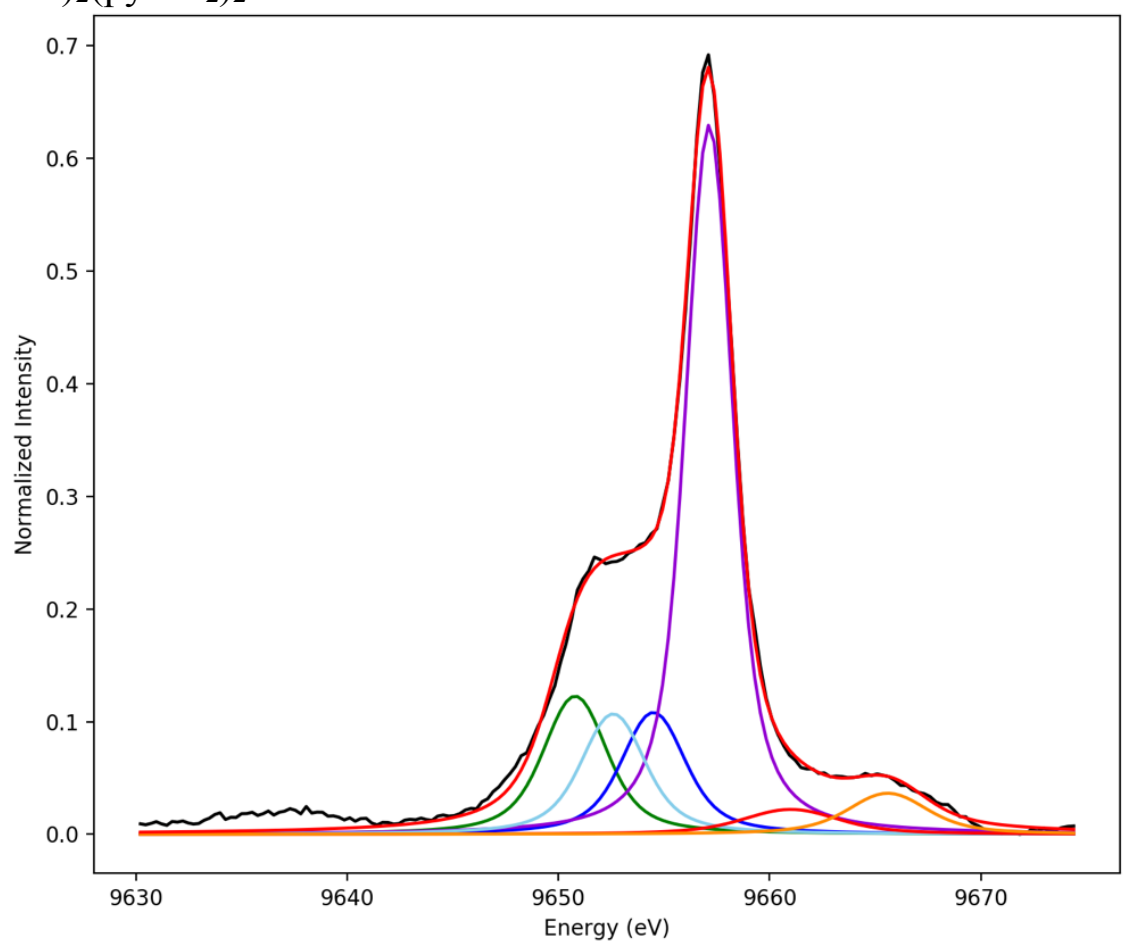


c. $\mathrm{Zn}(\mathrm{SPh})_{4}$

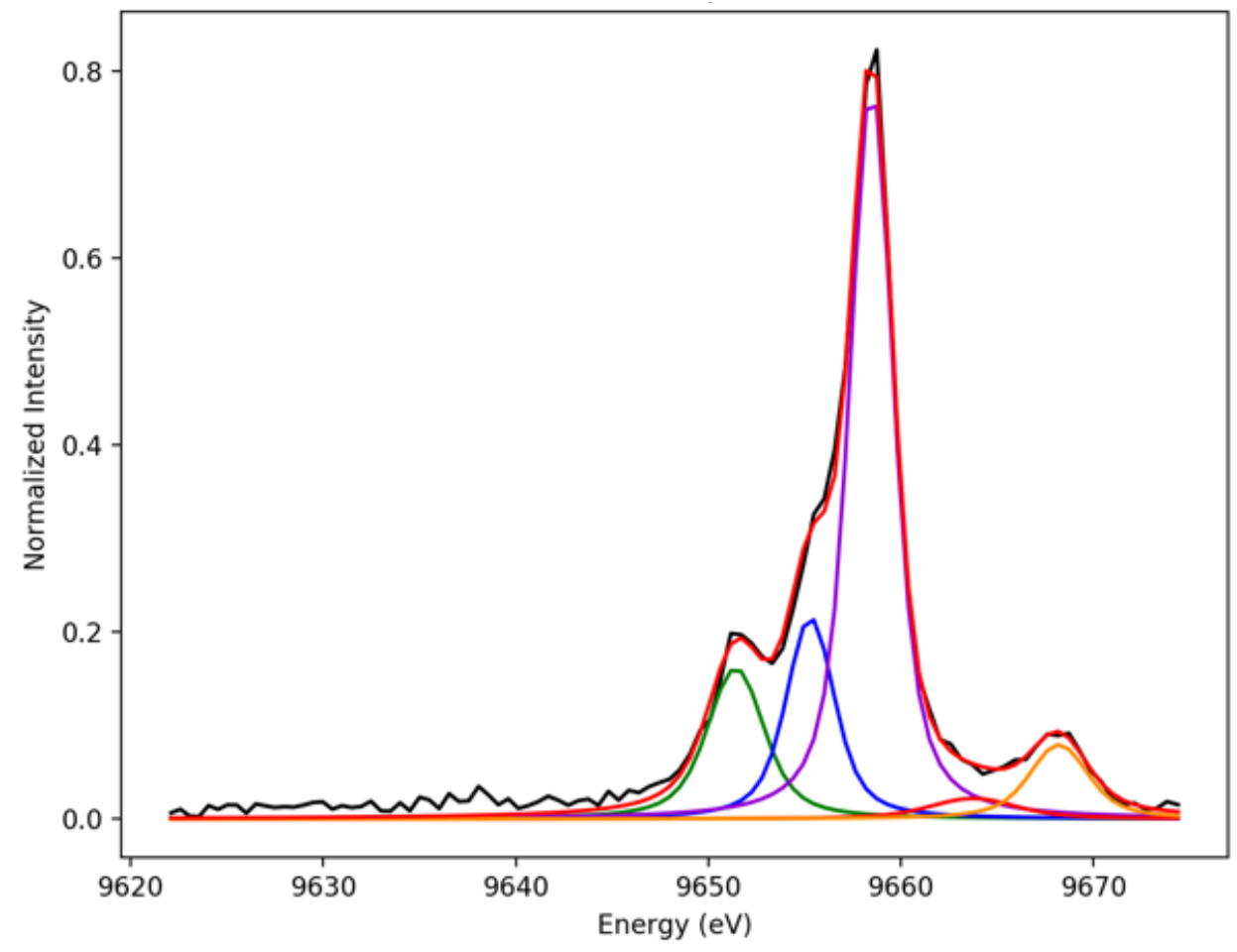

d. $\mathrm{Zn}\left({ }^{\mathrm{Me}} \mathrm{Im}\right)_{2}(\mathrm{SPh})_{2}$

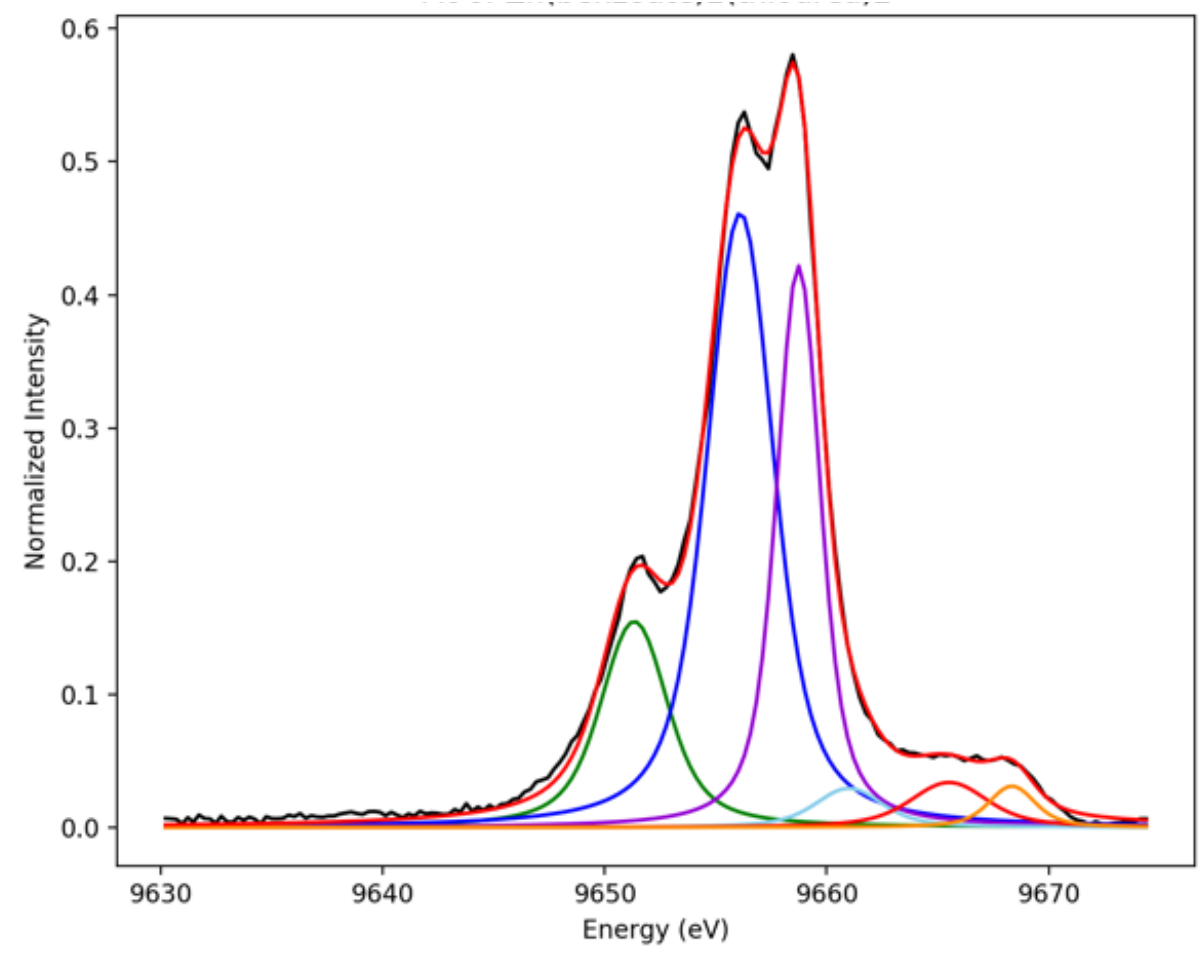


e. $\mathrm{Zn}\left({ }^{\mathrm{Me}} \mathrm{Im}\right)_{4}$

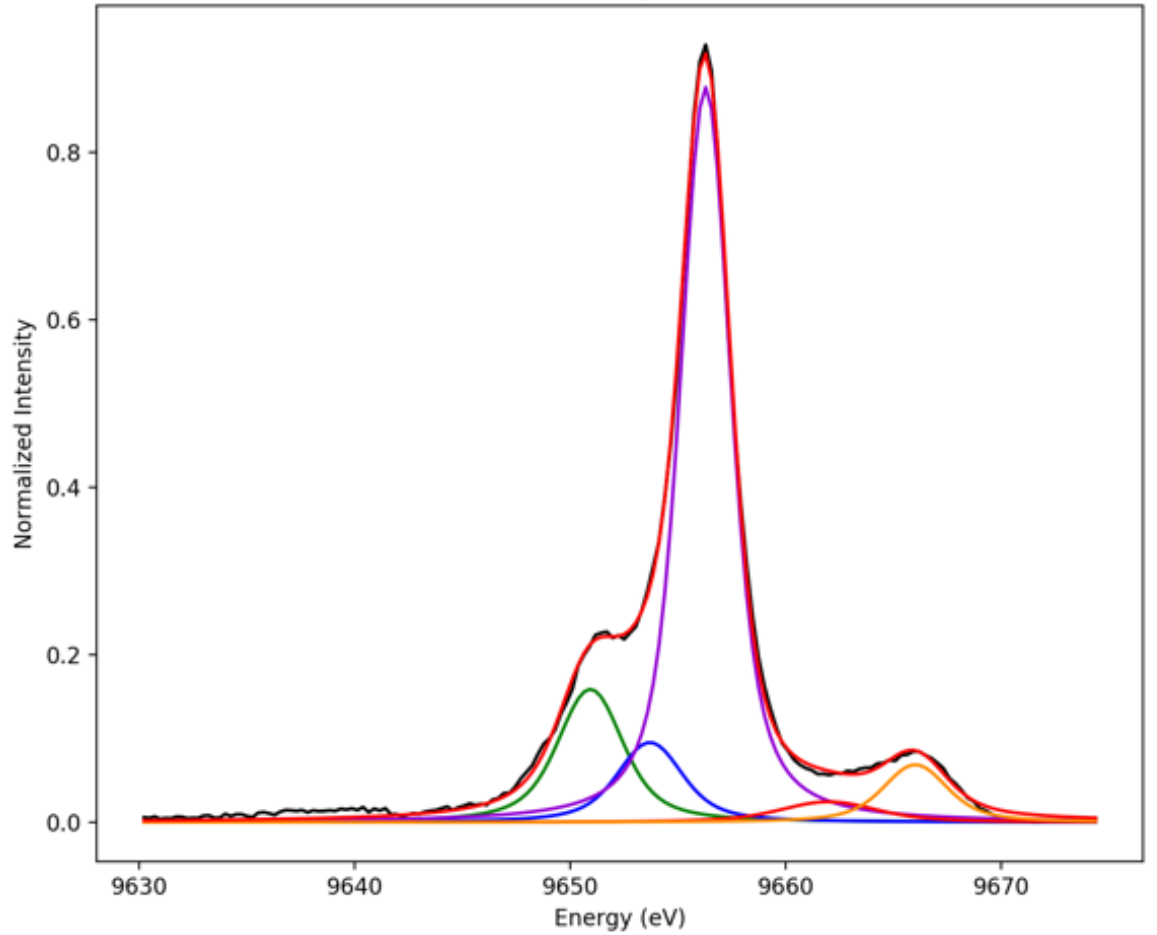

f. $\mathrm{Zn}\left({ }^{\mathrm{Me}} \mathrm{Im}\right)_{6}$

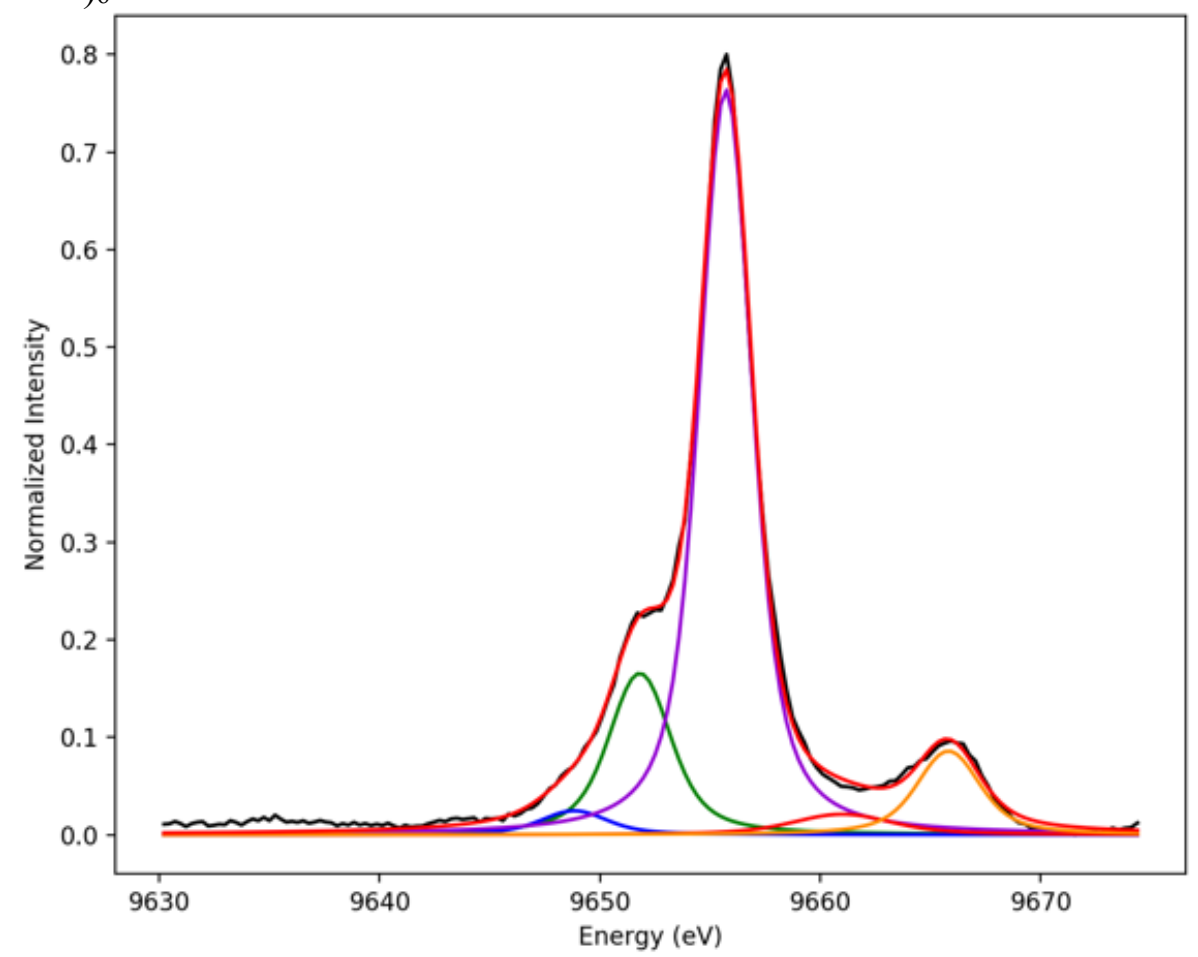

Figure S2. Fits to experimental VtC XES data 


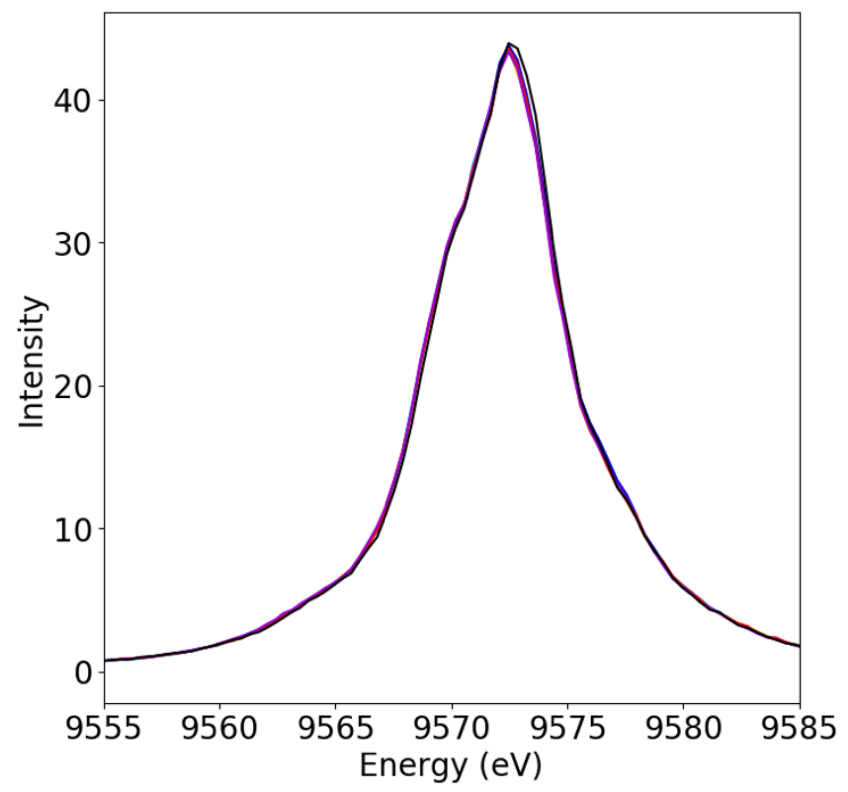

Figure S3. Overlay of $\mathrm{K} \beta$ mainline region for all measured samples. 
a. Molecular model of geometry optimized $\mathrm{Zn}\left({ }^{\mathrm{Me}} \mathrm{Im}\right)_{2}(\mathrm{SPh})_{2}$ with the angle of interest displayed

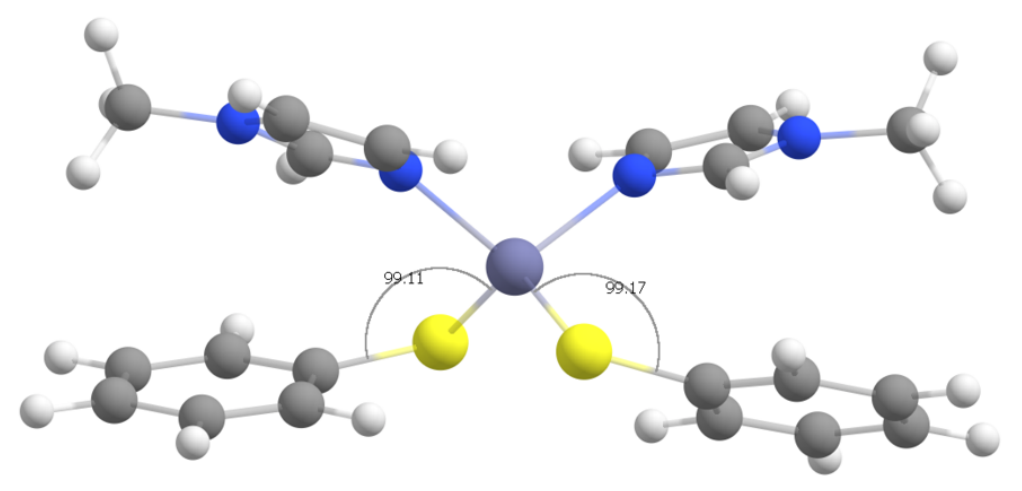

b. The effect of modulating the displayed angle on the $\mathrm{VtC}$ spectra, with the spectra of geometry optimized $\mathrm{Zn}\left({ }^{\mathrm{Me}} \mathrm{Im}\right)_{2}(\mathrm{SPh})_{2}$ and the average of the geometry optimized $\mathrm{Zn}\left({ }^{\mathrm{Me}} \mathrm{Im}\right)_{4}$ and $\mathrm{Zn}(\mathrm{SPh})_{4}$ for reference.

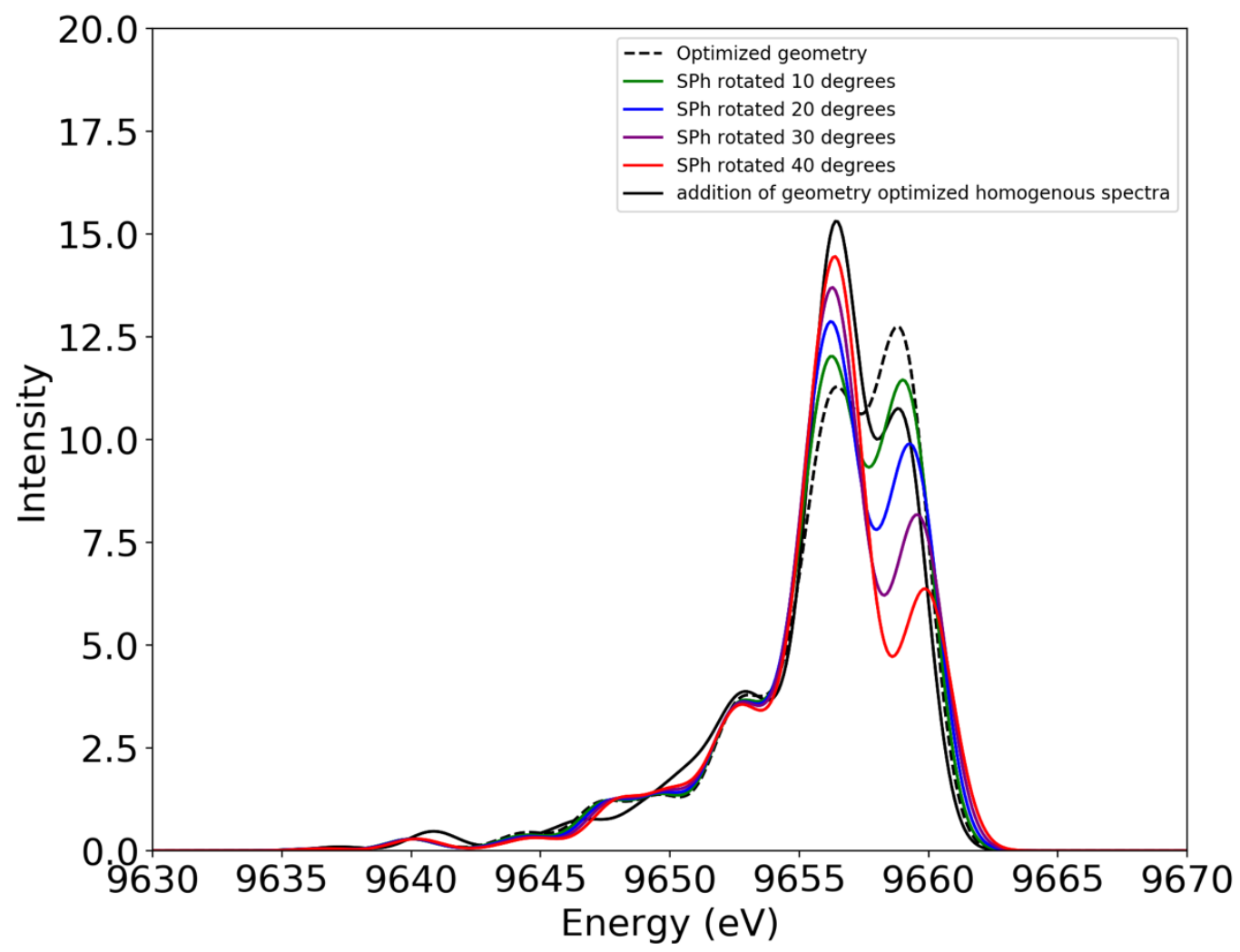

Figure S4. Zn( $\left.{ }^{\mathrm{Me}} \mathrm{Im}\right)_{2}(\mathrm{SPh})_{2}$ Ligand-Ligand Interaction Calculations 